\title{
Arteritis de células gigantes
}

\author{
J. Ma . CALVO ROMERO
}

Servicio de Medicina Interna. Hospital Regional Universitario Infanta Cristina. Badajoz

\author{
GIANT CELL ARTERITIS
}

\section{RESUMEN}

La arteritis de células gigantes (ACG) o arteritis temporal es la vasculitis sistémica más frecuente en nuestro medio, y su incidencia puede haber aumentado en los últimos años. La ACG afecta a vasos de mediano y grueso calibre, con preferencia por las arterias extracraneales, y ocurre en mayores de 50 años. La etiopatogenia es desconocida, aunque diversos agentes infecciosos pueden estar implicados. Una biopsia de arteria temporal normal no excluye el diagnóstico de ACG, ya que las lesiones pueden ser segmentarias. Las complicaciones más temibles de la ACG son la pérdida visual permanente, los ictus isquémicos y los aneurismas de aorta torácica y abdominal. El tratamiento consiste en corticoides a dosis altas, y actualmente no existe una alternativa terapéutica eficaz sin efectos adversos importantes. La mortalidad de los pacientes con ACG tratados no parece estar aumentada significativamente, probablemente debido al correcto diagnóstico y manejo de esta entidad.

PALABRAS CLAVE: Arteritis de células gigantes. Arteritis temporal.

\begin{abstract}
Giant cell arteritis (GCA) or temporal arteritis is the most frequent systemic vasculitis in our area, and its incidence may be on the increase in the last years. GCA involves large and medium-sized vessels, with pre ference to the extracranial arteries, and it affects persons older than 50 years. The aetiopathogenesis is unknown, although several infectious agents may be implicated. A normal temporal artery biopsy do not exclu de a GCA since the lesions may be skip. The most feared complications of GCA are permanent visual loss, ischemic strokes and thoracic and abdominal aortic aneurysms. The treatment consists of high-dose corti coids, and nowadays there is no effective therapeutic alternative without important adverse effects. The mortality of treated patients with GCA does not seem to be increased, probably due to a correct diagnosis and management of this entity.
\end{abstract}

KEY WORDS: Giant cell arteritis. Temporal arteritis.

Calvo Romero JM ${ }^{a}$. Arteritis de células gigantes. An Med Interna (Madrid) 2002; 19: 257-262.

\section{INTRODUCCIÓN}

La arteritis de células gigantes (ACG) o arteritis temporal es la vasculitis sistémica más frecuente en nuestro medio (1). Se presenta casi exclusivamente en mayores de 50 años, afecta a vasos de mediano y grueso calibre, con preferencia por las arterias extracraneales, y el diagnóstico de certeza requiere la realización de una biopsia de la arteria temporal (1-14). La ACG puede ser causa de pérdida visual permanente y de ictus isquémicos, entre otras complicaciones (114). El conocimiento de esta entidad permite un diagnóstico precoz y la instauración de un tratamiento adecuado para evitar dichas complicaciones. En el presente trabajo se revisan la epidemiología, etiopatogenia, manifestaciones clínicas, complicaciones, diagnóstico, tratamiento y pronóstico de la ACG.

\section{EPIDEMIOLOGÍA Y ETIOPATOGENIA}

La ACG es la vasculitis sistémica más frecuente en países occidentales (1-5). En la tabla I se describe la incidencia de la ACG en distintas zonas geográficas. En el norte de Europa y Norteamérica, la incidencia de ACG es mayor que en el área mediterránea $(1,4,5,8,9,11,12)$. La incidencia estimada en nuestro país es similar a la de países de nuestro entorno, 11 casos por 100.000 habitantes y año en mayores de 50 años (1). Parece que la incidencia de ACG puede haber aumentado en nuestro medio y en otras zonas geográficas en las últimas décadas (1012). En un estudio en un área de Galicia, la incidencia de ACG en mayores de 50 años aumentó de 8,26 a 10,49 casos por 100.000 habitantes y año en los periodos 1986-1990 y 19911995, respectivamente (10). La ACG se presenta con igual frecuencia en ambos sexos o algo más frecuentemente en la mujer,

Trabajo aceptado: 9 de julio de 2001

Correspondencia: José María Calvo Romero. Héroes de Cascorro, $93^{\circ} \mathrm{A} .06004$ Badajoz. 


\section{TABLA I}

INCIDENCIA DE LA ACG EN MAYORES DE 50 AÑOS EN DISTINTAS ZONAS GEO GRÁFICAS

\begin{tabular}{lcc}
\hline & Años & Incidencia \\
\hline Noruega (Aust Agder) (4) & $1987-94$ & 29 \\
Islandia (7) & $1984-90$ & 27 \\
Suecia (Goteborg) (5) & $1973-75$ & 18 \\
Estados Unidos (M inesota) (11) & $1950-85$ & 17 \\
España (Lugo) (1) & $1988-97$ & 11 \\
Israel (Jerusalen) (12) & $1980-91$ & 10 \\
Francia (Loire-Atlantique) (9) & $1970-79$ & 9 \\
Italia (Reggio Emilia) (8) & $1980-88$ & 7
\end{tabular}

ACG: arteritis de células gigantes. Incidencia por 100.000 habitantes $y$ año.

y afecta casi exclusivamente a mayores de 50 años (1-14). Por ejemplo, en nuestra serie de pacientes diagnosticados de ACG en la última década, el $64 \%$ fueron mujeres y la edad media al diagnóstico fue 72 años (13).

La etiopatogenia de la ACG es desconocida (3). Se ha propuesto que un insulto de naturaleza inmune en la pared vascular iniciaría una reacción en la arteria que llevaría a cambios estructurales, hiperplasia de la íntima y oclusión de la luz arterial (3). Este proceso parece depender de los linfocitos $\mathrm{T}$ presentes en la adventicia, que producen citoquinas, y las células efectoras serían los macrófagos y las células gigantes que se encuentran bajo el control de estos linfocitos T (3). La atrofia y la calcificación de la pared arterial parecen ser requisitos previos para la evolución del proceso inflamatorio, por lo que se supone que dicho proceso se inicia como una reacción focal de células gigantes frente a cuerpo extraño que posteriormente da lugar a una inflamación mas difusa y extensa (15). El papel de diversos agentes infecciosos como desencadenantes de la ACG es controvertido. La existencia de patrones cíclicos en la incidencia de ACG apoya la hipótesis de una causa infecciosa $(16,17)$. En Dinamarca se ha descrito la ocurrencia de varios picos de incidencia de ACG coincidentes con epidemias de infección por Mycoplasma pneumoniae, Chlamydia pneumoniae y parvovirus B19 (17). Algunos autores han detectado el DNA de Chlamydia pneumoniae en las muestras de arteria temporal de pacientes con ACG (18), mientras que otros no han podido confirmar este hallazgo (19). Otros estudios también han implicado al virus parinfluenza y al parvovirus B19 $(20,21)$. Diversos patrones en la producción de citoquinas pueden ser responsables de diferentes formas de presentación de la ACG (22). Los pacientes con ACG presentan mayor frecuencia de determinados antígenos del sistema HLA, lo que sugiere la existencia de una susceptibilidad genética $(8,23)$. En resumen, se supone que la ACG se debe a una respuesta inflamatoria celular que podría ser desencadenada por agentes externos, posiblemente infecciosos, en pacientes genéticamente predispuestos (3).

\section{MANIFESTACIONES CLÍNICAS Y COMPLICACIONES}

Las manifestaciones clínicas en 2 grandes series españolas de pacientes con ACG se resumen en la tabla II. El tiempo de
TABLA II

CARACTERÍSTICAS DE LA ACG EN 2 GRANDES SERIES ESPAÑ O LAS

\begin{tabular}{lcc}
\hline & $\begin{array}{c}\text { González-Gay y } \\
\text { García-Porrúa (1) }\end{array}$ & $\begin{array}{c}\text { Armona } \\
\text { et al (6) }\end{array}$ \\
\hline Número de casos & 110 & 191 \\
Sexo (mujer) & $50 \%$ & $53 \%$ \\
Edad media (años) & ND & 73 \\
Cefalea & $84 \%$ & $87 \%$ \\
Fiebre/ febrícula & ND & $29 \%$ \\
Síndrome constitucional & ND & $14 \%$ \\
Arterias temporales anormales & $65 \%$ & $75 \%$ \\
Claudicación mandibular & $43 \%$ & $40 \%$ \\
Amaurosis fugax & $13 \%$ & $25 \%$ \\
Amaurosis irreversible & $15 \%$ & $12 \%$ \\
Polimialgia reumática & $42 \%$ & $49 \%$ \\
\hline
\end{tabular}

ACG: arteritis de células gigantes. ND: dato no descrito

evolución de la clínica hasta el diagnóstico es bastante variable, y depende fundamentalmente de la expresividad clínica del cuadro y de la sospecha clínica de esta entidad (1-14). Casi todos los pacientes con ACG presentan una cefalea de reciente inicio o de características distintas a cefaleas previas (1-14). Manifestaciones sistémicas como fiebre o febrícula, anorexia y pérdida de peso ocurren con frecuencia variable (114). La fiebre de origen desconocido es una forma de presentación de la ACG, aunque no frecuente $(3,7)$.

La afectación arterítica en la ACG es objetivada con más frecuencia en las arterias extracraneales. Un hallazgo posible pero de frecuencia muy variable en la exploración física es la presencia de unas arterias temporales anormales a la palpación (ausencia o disminución del pulso, dolor, engrosamiento, induración y/o presencia de nódulos) (1-14). Hay que tener en cuenta que la ausencia o disminución del pulso de las arterias temporales también puede ser debida a aterosclerosis (14). Estas alteraciones también pueden encontrarse en la exploración de las arterias auricular posterior, facial y occipital $(14,24)$. Puede existir dolor en las arterias carótidas (1-14). La claudicación mandibular consiste en la aparición de dolor y/o fatigabilidad en los músculos faciales que se desencadenan por la masticación y que se alivian con el reposo (25). La claudicación mandibular se debe a afectación arterítica de la arteria maxilar inferior y facial y de sus ramas, es bastante característica de la ACG, pero sólo está presente en menos de la mitad de los casos $(1,6,7)$. No obstante, la claudicación mandibular también ocurre en otras vasculitis, en la ateroesclerosis, en la trombosis de la arteria carótida externa y en la amiloidosis sistémica (25-27). Hay casos de ACG con afectación de la arteria lingual, con dolor y necrosis de la lengua, e incluso dolor dentario $(1,29)$. También se ha descrito la necrosis del cuero cabelludo (30). La hipoacusia neurosensorial es otra posible manifestación de la ACG, aunque muy infrecuente (1,5\% en la serie de Hausch y Harrington) (31).

La neuropatía óptica isquémica es la complicación ocular más temida de la ACG, y se debe a la oclusión de la arteria oftálmica, arterias ciliares posteriores, arterias corodiales, arte- 
rias nutrientes del nervio óptico o arteria central de la retina (32-34). También puede ocurrir, pero menos frecuentemente, una trombosis de la arteria central de la retina (32-34). La presentación clásica consiste en un deterioro súbito, indoloro y monocular de la visión (32-34). La pérdida visual puede ser también bilateral, e ir precedida o no de amaurosis fugax (3234). Otra posible manifestación visual de la ACG es la diplopía (32-34). La pérdida visual permanente ocurre todavía en un 7 $15 \%$ de los pacientes (32-34). En una serie española de las 2 últimas décadas de 161 pacientes con $\mathrm{ACG}$, el $26 \%$ presentaron complicaciones isquémicas visuales y el $15 \%$ amaurosis irreversible (debida fundamentalmente a neuropatía óptica isquémica anterior y frecuentemente precedida de amaurosis fugax) (34). Los factores de riesgo para amaurosis irreversible más importantes son la presencia de amaurosis fugax e ictus isquémicos; y por el contrario, la presencia de anemia y marcadores de inflamación se asocia a un riesgo menor $(34,35)$. La aparición de pérdida visual o su progresión es muy infrecuente una vez iniciado el tratamiento con corticoides (33).

La ACG puede afectar a la circulación vértebro-basilar y carotídea (36). En un estudio del Reino Unido, 2 pacientes $(0,8 \%)$ de un total de 244 pacientes con un ictus isquémico presentaron una ACG (37). La ACG puede causar infarto agudo de miocardio debido a arteritis coronaria (38), pero la frecuencia con que ocurre es desconocida. La ACG se asocia a un riesgo aumentado de aneurismas de aorta torácica y abdominal y disección aórtica, debido al desarrollo de aortitis (39-41). También puede existir insuficiencia aórtica (39-41). En el 11,5\% y 5\% de una serie de pacientes con ACG se documentó la existencia de aneurismas de aorta torácica y abdominal, respectivamente (40). El riesgo de aneurisma de aorta torácica está aumentado unas 17 veces en los pacientes con ACG, y el de aneurisma de aorta abdominal unas 2,4 veces (40). La afectación de grandes arterias puede ser la forma de presentación de la ACG (41). En las arterias de las extremidades, más frecuentemente en miembros superiores que en miembros inferiores, puede también objetivarse arteritis en la ACG (41). La ACG con afectación de grandes arterias cursa sin afectación de las arterias extracraneales en un tanto por ciento significativo de pacientes, y puede constituir una variante específica de la ACG (42).

La frecuencia de complicaciones isquémicas en pacientes con ACG en nuestro medio se ha estimado en el $16 \%$ (35). Los pacientes con complicaciones isquémicas presentan con menor frecuencia fiebre y pérdida de peso, y tienen una velocidad de sedimentación globular (VSG) menos elevada y una concentración de hemoglobina mayor $(34,35)$. La presencia de datos clínicos y analíticos indicativos de una fuerte respuesta inflamatoria parece pues que disminuye el riesgo de complicaciones isquémicas en la ACG $(34,35)$.

La polimialgia reumática (PMR) es un síndrome clínico de etiología desconocida caracterizado por rigidez y dolor cervical y en cinturas escapular y pelviana, que está presente en un tanto por ciento variable de casos de ACG (43). La mayoría de los autores considera que existen suficientes diferencias entre la ACG y la PMR para considerarlas entidades distintas (43). La existencia de distintos patrones de antígenos del sistema HLA en la ACG y la PMR apoya este concepto (21). Por otro lado, se ha descrito la producción de citoquinas en las muestras de arteria temporal de pacientes con PMR sin ACG asociada, sugiriendo que en estos pacientes podría existir ya una vasculitis "silente", y apoyando la idea de que la PMR y la ACG son presentaciones clínicas dentro del espectro de una misma entidad $(44,45)$. Un $64 \%$ de nuestra serie de casos de ACG presentó PMR (13), frecuencia superior a la de otras series españolas (Tabla II). En el norte de Italia, la frecuencia de PMR en la ACG es del 46\% (8), y en otros estudios de Holanda y Canadá del $36 \%$ y $56 \%$, respectivamente $(46,47)$. Esta variabilidad en la frecuencia de PMR en la ACG puede deberse, entre otros factores, a la existencia de diferencias geográficas, a la inclusión en algunos estudios de pacientes sin confirmación histológica de ACG y a la mayor o menor rigurosidad de los criterios utilizados para establecer el diagnóstico de PMR. La ACG con PMR asociada no difiere sustancialmente en sus características de la ACG sin PMR (48).

La elevación de la VSG es casi constante en la ACG (114), si bien ocasionalmente puede ser normal o estar sólo ligeramente elevada $(49,50)$. En una serie española, un 5\% de los casos de ACG tuvieron una VSG inferior a $50 \mathrm{~mm} / \mathrm{hora}(6)$. Otros reactantes de fase aguda como la proteína $\mathrm{C}$ reactiva y el fibrinógeno también están habitualmente elevados $(3,13,14)$. La anemia es habitualmente moderada, y está presente en la mitad de los casos de ACG $(3,13,14)$. En torno a un tercio de los pacientes con ACG presentan trombocitosis $(3,13,14,51)$. En pacientes con complicaciones isquémicas de la ACG el recuento plaquetario es mayor, lo que puede ser debido a una respuesta de fase aguda reflejando una mayor severidad de la vasculitis o a que la trombocitosis participe en la etiopatogenia de las complicaciones isquémicas de la ACG (51). Aproximadamente un tercio de los pacientes con ACG tienen elevación de las enzimas hepáticas, siendo más frecuente la elevación de la fosfatasa alcalina $(3,13,14)$.

Debe mencionarse que en los últimos años se ha descrito una disminución de la frecuencia de manifestaciones clínicas clásicas de la ACG como síndrome constitucional, PMR, cefalea, arterias temporales anormales a la exploración, amaut rosis fugax, y de alteraciones analíticas típicas (anemia, trombocitosis y elevación de la fosfatasa alcalina) (10).

\section{DIAGNÓSTICO}

La confirmación del diagnóstico de ACG requiere la realización de una biopsia de la arteria temporal $(3,14)$. La ACG se caracteriza por la presencia de un infiltrado inflamatorio de linfocitos y macrófagos en la pared arterial, necrosis de la lámina elástica interna, células gigantes multinucleadas y granulomas $(3,14)$. La presencia de células gigantes multinucleadas y granulomas no es imprescindible para poder establecer el diagnóstico de ACG $(3,14)$. La biopsia de la arteria temporal es una técnica sencilla, poco costosa $y$ con escasas o nulas complicaciones $(3,14,52)$. Una biopsia de arteria temporal normal no excluye el diagnóstico de ACG $(3,14)$. La afectación de la arteria temporal puede ser segmentaria por lo que en ocasiones la biopsia puede ser negativa. La sensibilidad de la biopsia de la arteria temporal es muy variable (38-90\%) según las series, y aumenta con la longitud de la muestra de arteria $(3,14,53)$. Los falsos negativos se pueden minimizar si se toma una muestra de 3-4 centímetros, y si el resultado es negativo realizando biopsia de la arteria temporal contralateral $(3,14)$. El examen histológico rutinario a múltiples niveles no parece aumentar el rendimiento diagnóstico de la biopsia de arteria temporal, aunque en determinados casos puede estar justificado (54). El tanto por ciento de biopsias de arteria temporal positivas es muy variable, y depende de los criterios utilizados para 
su indicación, de la longitud de la muestra de arteria temporal y de la realización o no de biopsia contralateral en los casos con una primera biopsia negativa $(55,56)$. Gabriel et al. identificaron un grupo de pacientes con una alta probabilidad de tener una biopsia de arteria temporal negativa, que corresponde a pacientes sin claudicación mandibular, con una exploración normal de las arterias temporales, con sinovitis y con una VSG sólo discretamente elevada (57). En base a esto, la biopsia de la arteria temporal podría ser inicialmente evitada en estos pacientes con muy bajo riesgo de ACG, y se debería insistir en la búsqueda de un diagnóstico alternativo.

El American College of Rheumatology (ACR) definió en 1990 unos criterios para la clasificación de la ACG (Tabla III) (58). La presencia de 3 de estos 5 criterios tiene una sensibilidad y una especificidad para el diagnóstico de ACG del 93 y $91 \%$, respectivamente (59). Estos criterios de la ACR han sido validados en España (6). No obstante, la mayoría de los autores aceptan que la biopsia de arteria temporal debe realizarse para confirmar el diagnóstico, teniendo en cuenta los potenciales efectos adversos de la corticoterapia a altas dosis necesaria para el tratamiento de la $\operatorname{ACG}(3,14,55,56)$.

\section{TABLA III}

\section{CRITERIOS PARA LA CLASIFICACIÓN DE LA ACG DEL AM ERICAN CO LLEGE O F RHEUM ATO LOGY}

Edad igual o superior a 50 años

Cefalea localizada de reciente inicio

Arterias temporales dolorosas o con pulso disminuido

VSG igual o superior a $50 \mathrm{~mm} /$ hora

Biopsia positiva*

VSG: velocidad de sedimentación globular. *Biopsia arterial con arteritis necrotizante caracterizada por infiltrado mononuclear y/o granulomas

Un tema de discusión es si debe realizarse biopsia de arteria temporal en todos los pacientes con PMR. Rodríguez Valverde et al. describieron tres grupos de alto riesgo de ACG en pacientes con PMR: 1) mayores de 70 años con cefalea de reciente inicio y arterias temporales anormales a la exploración; 2) pacientes con cefalea de reciente inicio, claudicación mandibular y arterias temporales anormales a la exploración; y 3$)$ pacientes con las características del grupo b y edad superior a 70 años (59). Los pacientes de menos de 70 años, sin cefalea, y con arterias temporales normales a la exploración constituyeron un grupo de muy bajo riesgo de ACG, ya que sólo 1 caso $(1,6 \%)$ de un total de 59 pacientes con PMR con estas características presentó ACG (59).

Los linfocitos predominantes en el infiltrado inflamatorio de la ACG son los CD4 (60), lo que explica que un recuento de linfocitos CD8 disminuido en sangre periférica pueda tener valor diagnóstico en la ACG (61). La ecografía-doppler de las arterias temporales puede demostrar signos de vasculitis (estenosis y un halo hipoecoico debido a edema de la pared arterial) en la ACG (62). La mayoría de los pacientes con ACG presentan una disminución de la velocidad del flujo sanguíneo en la arteria temporal superficial determinada mediante ecografía-doppler con color (63).
Una captación gammagráfica de galio-67 elevada en la región temporal apoya el diagnóstico de ACG, incluso en pacientes con biopsia de arteria temporal negativa, con un valor predictivo positivo y una especificidad del 90 y $94 \%$, respectivamente (64). Además la remisión de la ACG se acompaña de una normalización de la captación de galio (64).

\section{TRATAMIENTO Y PRONÓSTICO}

El tratamiento de la ACG requiere corticoides a dosis elevadas $(3,14,65)$. La dosis inicial debería ser de 40-60 mg de prednisona al día o su equivalente $(3,14,65)$. La respuesta suele ser espectacular en 48-72 horas, con resolución de las manifestaciones clínicas $(3,14)$. Ante la sospecha de ACG debe iniciarse corticoterapia inmediatamente debido al riesgo de pérdida visual permanente y otras complicaciones irreversibles $(3,14,65)$. Además, el resultado de la biopsia de la arteria temporal no se modifica sensiblemente incluso dos semanas después de iniciada la corticoterapia, aunque puedan desaparecer algunas características histológicas típicas (66). En pacientes que presentan ya alteraciones visuales u otras complicaciones isquémicas algunos autores recomiendan administrar dosis iniciales altas de corticoides, por ejemplo $500 \mathrm{mg}$ de metilprednisolona intravenosa $(3,14)$. No obstante, otros autores dudan de la utilidad de esta recomendación (67). Se ha sugerido en base a observaciones aisladas que el inicio del tratamiento con corticoides en la ACG puede estar relacionado con la ocurrencia de ictus isquémicos, muchos de ellos progresivos y de localización vértebro-basilar (68).

No existe acuerdo en el tiempo que debe prolongarse la corticoterapia $(3,14)$. El protocolo terapéutico debe ser siempre individualizado, y los pacientes deben ser revisados regularmente. Una gran parte de los pacientes con ACG requieren corticoterapia de mantenimiento a dosis bajas durante 2-3 años $(3,14,69)$. Casi el $90 \%$ de los pacientes de una serie tuvieron una ACG inactiva tras un seguimiento medio de 7,1 años, aunque aproximadamente un tercio todavía recibía corticoterapia (70). La dosis de corticoides generalmente es reducida al mes si las manifestaciones clínicas han desaparecido y la VSG y la proteína $\mathrm{C}$ reactiva se han normalizado $(3,14,69)$. La reducción debe ser gradual, y a partir de $10 \mathrm{mg} /$ día disminuir la dosis de prednisona $1 \mathrm{mg}$ cada $2-4$ semanas $(3,14,69)$. Hasta el $60 \%$ de los pacientes pueden presentar exacerbaciones en relación con la reducción de la corticoterapia (71). La monitorización debe basarse en la clínica y en reactantes de fase aguda como la VSG y la proteína C reactiva $(3,14,69)$. Una elevación aislada de la VSG no obliga generalmente a subir la dosis de corticoides $(3,14,69)$. La interleucina-6 puede ser más sensible que la VSG como indicador de actividad en la ACG. En un reciente estudio de pacientes con ACG, la interleucina-6 estuvo elevada en el $89 \%$ de las recurrencias, mientras que la VSG sólo estuvo elevada en el $58 \%$ de las recurrencias (71). La disminución de la concentración sérica de interleucina- 6 con la corticoterapia coincide generalmente con la resolución de las manifestaciones clínicas de la ACG (71). Casi un 50\% de los pacientes con ACG pueden tener anticuerpos anticardiolipina a títulos iguales o superiores a 20 $\mathrm{U}$ (72). Estos anticuerpos anticardiolipina disminuyen con la corticoterapia, y se elevan hasta en el $74 \%$ de las exacerbaciones de la ACG, y no se elevan por otros procesos intercu- 
rrentes como infecciones (72). No obstante, son necesarios estudios adicionales para poder recomendar el uso rutinario de la interleucina- 6 y de los anticuerpos anticardiolipina en la monitorización de la actividad de la ACG.

Debido al riesgo de osteoporosis y fracturas, en todos los pacientes con ACG que reciben corticoterapia a largo plazo se recomienda la administración de suplementos de calcio y vitamina D, y en los pacientes con densitometría patológica la administración de bifosfonatos $(3,14)$. El metotrexato se ha propuesto como alternativa para reducir la dosis de corticoides en la ACG, aunque los resultados han sido contradictorios (73-75). En casos de ACG con escasa respuesta a los corticoides o en los que se intente reducir la dosis de corticoides debido a sus efectos adversos, la azatioprina o la dapsona pueden ser otras opciones $(75,76)$. Algunos estudios han sugerido que la ACG puede ser más severa en el sexo femenino, lo que podría tener implicaciones terapéuticas $(6,13)$.

Es de esperar que en un futuro próximo se realicen ensayos clínicos con inmunosupresores u otros fármacos que permitan reducir las dosis de corticoides necesarias en la ACG y minimizar de esta forma sus efectos adversos. Así por ejemplo, fármacos capaces de suprimir la producción de interferón-gamma pueden ser útiles en el tratamiento de la ACG, ya que el interferón-gamma parece tener un papel etiopatogénico determinante en la ACG $(44,77)$.
La mortalidad de la ACG en nuestro medio es baja, posiblemente debido al mayor conocimiento de la enfermedad, al diagnóstico precoz, al correcto tratamiento y adecuado seguimiento (78). La esperanza de vida en pacientes con ACG no difiere significativamente de la población general de igual edad $(70,78,79)$. No obstante, las complicaciones vasculares parecen ser una causa importante de muerte en pacientes con ACG en los primeros meses tras el diagnóstico. En un estudio de 284 pacientes, casi el $6 \%$ fallecieron en los 4 primeros meses, fundamentalmente de enfermedad cerebrovascular o infarto agudo de miocardio (79). A un plazo de 3 años existe una elevada frecuencia de complicaciones relacionadas con la corticoterapia, especialmente infecciones severas y fracturas, e incluso una mortalidad aumentada debida a estas complicaciones, sobre todo en mayores de 75 años con corticoterapia prolongada (80).

Las neoplasias pueden presentarse con síntomas similares a la ACG $(81,82)$. La persistencia de los síntomas de ACG y/o la no normalización de los reactantes de fase aguda con la corticoterapia debe hacer considerar otros diagnósticos, entre los que se incluyen fundamentalmente las neoplasias (81). En una serie de pacientes con ACG hubo un aumento de la incidencia de cáncer, aunque el largo intervalo de tiempo entre el diagnóstico de ambas entidades hace improbable que la ACG pueda ser en algunos casos un síndrome paraneoplásico (82).

\section{Bibliografía}

1. González-Gay MA, García-Porrúa C. Systemic vasculitis in adults in northwestern Spain, 1988-1997: Clinical and epidemiologic aspects. Medicine (Baltimore) 1999; 78:292-308.

2. Huston KA, Hunder GG, Lie JT, Kennedy RH, Elveback LR. Temporal arteritis. A 25 year epidemiological, clinical and pathologic study. Ann Intern Med 1978; 88: 162-7.

3. Evans JM, Hunder GG. Polymyalgia rheumatica and giant cell arteritis. Rheum Dis Clin North Am 2000; 26:493-515.

4. Gran JT, Myklebust G. The incidence of polymyalgia rheumatica and temporal arteritis in the county of Aust Agder, South Norway: a prospective study 1987-1994. J Rheumatol 1997; 24:1739-43.

5. Bengtsson BA, Malmwall BE. The epidemiology of giant cell arteritis including temporal arteritis and polymyalgia rheumatica: incidences of different clinical presentations and eye complications. Arthritis Rheum 1981; 24: 899-904.

6. Armona J, Rodríguez-Valverde V, González-Gay MA, et al. Arteritis de células gigantes. Estudio de 191 pacientes. Med Clin (Barc) 1995; 105 : 734-7.

7. Baldursson O, Steinsson K, Bjornsson J, Lie JT. Giant cell arteritis in Iceland. An epidemiologic and histopathologic analysis. Arthritis Rheum 1994; 37: 1007-12.

8. Salvarani C, Machioni P, Zizzi F, et al. Epidemiologic and inmunogenetic aspects of polymyalgia rheumatica and giant cell arteritis in Northern Italy. Arthritis Rheum 1991; 34: 351-6.

9. Barrier J, Pion P, Massari R, Peltier P, Rojouan J, Grolleau JY. Approche épidémiologique de la maladie de Horton dans le départment de Loire-Atlantique: 110 cas en 10 ans (1970-1979). Rev Med Interne 1983; 3 : 13-20.

10. González-Gay MA, Blanco R, Sánchez-Andrade A, Vázquez-Caruncho M. Giant cell arteritis in Lugo, Spain: a more frequent disease with fewer classic features. J Rheumatol 1997; 24: 2166-2170.

11. Machado EBV, Michet CJ, Ballard DJ, et al. Trends in incidence and clinical presentation of temporal arteritis in Olmsted County, Minnesota, 1950-1985. Arthritis Rheum 1988; 31: 745-9.

12. Sonnenblick M, Nesher G, Friedlander Y, Rubinow A. Giant cell arteritis in Jerusalem: A 12-year epidemiological study. Br J Rheumatol 1994; 33:938-41.

13. Calvo Romero JM, Magro Ledesma D, Ramos Salado JL, et al. Arteritis

de células gigantes: un estudio descriptivo en el suroeste de España. An Med Interna (Madrid) 2000; 17:67-70.

14. Swannell AJ. Polymyalgia rheumatia and temporal arteritis: diagnosis and treatment. BMJ 1997; 314:1329-32.

15. Nordborg C, Nordborg E, Petursdottir V. The pathogenesis of giant cell arteritis: morphological aspects. Clin Exp Rheumatol 2000; 18 (suppl 20): $18-21$

16. Salvarani C, Gabriel S, O'Fallon M, Hunder GG. The incidence of giant cell artertis in Olmsted County, Minnesota: apparent fluctuations in a cyclic pattern. Ann Intern Med 1995; 123: 192-4.

17. Elling P, Olsson AT, Elling H. Synchronous variations of the incidence of temporal arteritis and polymyalgia rheumatica in different regions of Denmark; association with epidemics of Mycoplasma penumoniae infection. J Rheumatol 1996; 23: 112-9.

18. Rimenti G, Blasi F, Cosentini R, et al. Temporal arteritis associated with Chlamydia pneumoniae DNA detected in an artery specimen. J Rheumatol 2000; 27: 2718-20.

19. Haugeberg G, Bie R, Nordbo SA. Chlamydia pneumoniae not detected in temporal artery biopsies from patients with temporal arteritis. Scand J Rheumatol 2000; 29: 127-8.

20. Duhaut P, Bosshard S, Calvet A, et al. Giant cell arteritis, polymyalgia rheumatica, and viral hypotheses: a multicenter, prospective case-control study. Groupe de Recherche sur l'Arterite a Cellules Geantes. J Rheumatol 1999; 26: 361-9.

21. Gabriel SE, Espy M, Erdman DD, Bjornsson J, Smith TF, Hunder GG. The role of parvovirus B19 in the pathogenesis of giant cell arteritis: a preliminary evaluation. Arthritis Rheum 1999; 42:1255-8.

22. Weyand CM, Tetzlaff N, Bjornsson J, Brack A, Youngue B, Gorozny JJ. Disease patterns and tissue cytokine profiles in giant cell arteritis. Arthritis Rheum 1997; 40: 19-26.

23. Dababneh A, González-Gay MA, García-Porrúa C, Hajeer A, Thomson W, Ollier W. Giant cell arteritis and polymyalgia rheumatica can be differentiated by distinct patterns of HLA class II association. J Rheumatol 1998; 25: 2140-5.

24. Achkar AA, Lie JT, Gabriel SE, Hunder GG. Giant cell arteritis involving the facial artery. J Rheumatol 1995; 22: 360-2.

25. Goodman BW, Shepard FA. Jaw claudication: its value as a diagnostic clue. Postgrad Med 1983; 73: 177-83 
26. Gertz MA, Kyle RA, Griffing WL, Hunder GG. Jaw claudication in primary systemic amyloidosis. Medicine 1986; 65: 173-9.

27. Venna W, Goldman R, Tilak S, Sabin TD. Temporal arteritis-like presentation of carotid atherosclerosis. Stroke 1986; 17: 325-7.

28. Vermuelen JP, Mahowald ML. A case of Wegener's granulomatosis presenting with jaw claudication. J Rheumatol 1984; 11: 707-9.

29. Patterson A, Scully C, Barnard N, Giffith MJ, Eveson JW, Novelli M. Necrosis of the tongue in a patient with intestinal infarction. Oral Surg Oral Med Oral Pathol 1992; 74: 582-6.

30. Sonderstrom CW, Seehafer JR. Bilateral scalp necrosis in temporal arteritis: a rare complication of Horton's disease. Am J Med 1976; 61: 541-6.

31. Hausch RC, Harrington T. Temporal arteritis and sensorineural hearing loss. Semin Arthritis Rheum 1998; 28: 206-9.

32. Tovilla-Canales JL. Ocular manifestations of giant cell arteritis. Curr Opin Ophthalmol 1998; 9: 73-9.

33. Aiello PD, Trautmann JC, McPhee TJ, Kunselman AR, Hunder GG.Visual prognosis in giant cell arteritis. Ophtalmology 1993; 100: 550-5.

34. González-Gay MA, García-Porrúa C, Llorca J, et al. Visual manifestations of giant cell arteritis. Trends and clinical spectrum in 161 patients. Medicine (Baltimore) 2000; 79:283-2.

35. Cid MC, Font C, Oristrell J, et al. Association between strong inflammatory response and low risk of developing visual loss and other cranial ischemic complications in giant cell (temporal) arteritis. Arthritis Rheum 1998; 41: 26-32.

36. Reich KA, Giansiracusa DF, Strogwater SL. Neurologic manifestations of giant cell arteritis. Am J Med 1990; 89: 67-72.

37. Sandercock PAG, Warlow CP, Jones LN, Starkey IR. Predisposing factors for cerebral infarction: the Oxfordshire community stroke project. BMJ 1989; 298: 75-80.

38. Paulley JW. Coronary ischaemia and occlusion in giant cell (temporal) arteritis. Acta Med Scand 1980: 208: 257-63.

39. Evans JM, Bowles CA, Bjornsson J, Mullany CJ, Hunder GG. Thoracic aortic aneurysm and rupture in giant cell arteritis. A descriptive study of 41 cases. Arthritis Rheum 1994; 37: 1539-47.

40. Evans JM, O'Fallon WM, Hunder GG. Increased incidence of aortic aneuysm and dissection in giant cell (temporal) arteritis. A population based study. Ann Intern Med 1995; 122: 502-7.

41. Lie JT. Aortic and extracranial large vessel giant cell arteritis. A review of 72 cases with histopathological documentation. Sem Arthritis Rheum 1995; 24: 422-31.

42. Brack A, Martínez-Taboada V, Stanson A, Gorozny JJ, Weyand CM. Disease pattern in cranial and large-vessel giant cell arteritis. Arthritis Rheum 1999; 42: 311-7.

43. Salvarani C, Machionni P, Boiardi L. Polymyalgia rheumatica. Lancet 1997; 350: 43-7.

44. Weyand CM, Hicok KC, Hunder GG, Gorozny JJ. Tissue cytokine patterns in patients with polymyalgia rheumatica and giant cell arteritis. Ann Intern Med 1994; 121: 484-91.

45. Hunder GG. Giant cell arterits in polymyalgia rheumatica. Am J Med 1997; 102: 514-6.

46. Mertens JCC, Willemsen G, Van Saase JLCM, Bolk JH, Dijkmans BAC. Polymyalgia rheumatica and temporal arteritis: a retrospective study of 111 patients. Clin Rheumatol 1995; 14: 650-5.

47. Bahlas S, Ramos-Remus C, Davis P. Clinical outcome of 149 patients with polymyalgia rheumatica and giant cell arteritis. J Rheumatol 1998; 25: 99-104.

48. González-Gay MA, García-Porrúa C, Vázquez-Caruncho M. Polymyalgia rheumatica in biopsy proven giant cell arteritis does not constitute a different subset but differs from isolated polymyalgia rheumatica. J Rheumatol 1998; 25: 1750-5.

49. Wong RL, Korn JH. Temporal arteritis without an elevated erythrocyte sedimentation rate. Am J Med 1986; 80: 959-64.

50. Martínez-Taboada VM, Blanco R, Arnona J, et al. Gaint cell arteritis with an erythrocyte sediemtation rate lower than 50. Clin Rheumatol 2000; 19: 73-5.

51. De Keyser J, De Klippel N, Ebinger G. Thrombocytois and ischaemic complications in giant cell arteritis. BMJ 1991; 303: 825.

52. Domínguez-Castellano A, Peña JM, Barbado FJ, et al. Utilidad de la biopsia de arteria temporal: análisis de 100 casos. Med Clin (Barc) 1989; 92: 85-8.

53. Sudlow C. Sensitivity of temporal artery biopsy varies with biopsy length and sectioning strategy (letter). BMJ 1997; 315: 549.

54. Chakrabarty A, Franks AJ. Temporal artery biopsy: is there any value in examining biopsies at multiple levels? J Clin Pathol 2000; 53: 131-6.

55. Hall S, Hunder GG. Is temporal artery biopsy prudent? Mayo Clin Proc 1984; 59: 793-6.

56. Martínez J, Carreño MC, Bilbao J, Román F, Pérez Maeztu R, Masa C. ¿Es necesaria la biopsia de la arteria temporal? Estudio de 204 biopsias.
Rev Clin Esp 1991; 189: 325-7.

57. Hunder GG, Bloch DA, Michel BA, et al. The American College of Rheumatology 1990 criteria for the classification of giant cell arteritis. Arthritis Rheum 1990; 33: 1122-8.

58. Gabriel SE, O'Fallon WM, Achkar AA, Lie JT, Hunder GG. The use of clinical characteristics to predict the results of temporal artery biopsy among patients with suspected giant cell arteritis. J Rheumatol 1995; 22 : 93-6.

59. Rodríguez-Valverde V, Sarabia JM, González-Gay MA, et al. Risk factors and predictive models of giant cell arteritis in polymyalgia rheumatica. Am J Med 1997; 102: 331-6.

60. Banks PM, Cohen MD, Ginsburg WW, Hunder GG. Immunohistologic and cytochemical studies of temporal arteritis. Arthritis Rheum 1983; 26: 1201-7.

61. Elling P, Olsson AT, Elling H. A reduced CD8+ lymphocyte subset distinguishes patients with polymyalgia rheumatica and temporal arteritis from patients with other diseases. Clin Exp Rheumatol 1998; 16 : 155-60.

62. Schmidt WA. Doppler ultrasonography in the diagnosis of giant cell arteritis. Clin Exp Rheumatol 2000; 18 (suppl 20): 40-2.

63. Lauwerys BR, Puttemans T, Houssiau FA, Devogelaer JP. Color doppler sonography of the temporal arteries in giant cell arteritis and polymyalgia rheumatica. J Rheumatol 1997; 24: 1570-4.

64. Genereau T, Lortholary O, Guillevin L, et al. Temporal 67gallium uptake is increased in temporal arteritis. Rheumatology (Oxford) 1999; 38: 709-13.

65. Kyle V, Hazleman BL. Treatment of polymyalgia rheumatica and giant cell arteritis. I. Steroid regimens in the first two months. Ann Rheum Dis 1989; 48: 658-61.

66. Achkar AA, Lie TJ, Hunder GG, O'Fallon WM, Gabriel SE. How does previous corticosteroid treatment affect the biopsy findings in giant cell (temporal) arteritis? Ann Intern Med 1994; 120: 987-92.

67. Cornblath WT, Eggenberger ER. Progressive visual loss from giant cell arteritis despite high-dose intravenous methylprednisolone. Ophthalmology 1997; 104: 854-8

68. Staunton H, Stafford F, Leader M, O'Riordain D Deterioration of giant cell arteritis with corticosteroid therapy. Arch Neurol 2000; 57: 581-4.

69. Ferraccioli GF, Poi ED, Damato R. Steroid sparing therapeutic approaches to polymyalgia rheumatica-giant cell arteritis. State of the art and perspectives. Clin Exp Rheumatol 2000; 18 (suppl 20): 58-60.

70. Matteson EL, Gold KN, Bloch DA, Hunder GG. Long-term survival of patients with giant cell arteritis in the American College of Rheumatology giant cell arteritis classification criteria cohort. Am J Med 1996; 100: 193-6.

71. Weyand CM, Fulbright JW, Hunder GG, Evans JM, Gorozny JJ. Treatment of giant cell arteritis: interleukin- 6 as a biologic marker of disease activity. Arthritis Rheum 2000; 43: 1041-8.

72. Liozon E, Roblot P, Paire D, et al. Anticardiolipin antibody levels predict flares and relapses in patients with giant cell (temporal) arteritis. A longitudinal study of 58 biopsy-proven cases. Rheumatology (Oxford) 2000; 39: 1089-94.

73. van der Veen MJ, Dinant HJ, Van Booma-Frankfort C, et al. Can methotrexate be used as a steroid sparing agent in the treatment of polymyalgia rheumatica and giant cell arteritis? Ann Rheum Dis 1996; 55: 218-23.

74. Hernandez-Garcia C, Soriano C, Morado C, et al. Methotrexate treatment in the management of giant cell arteritis. Scand J Rheumatol 1994; 23: $295-8$.

75. Nesher G, Sonnenblick M. Steroid sparing medications in temporal arteritis-report of three cases and review of 174 reported cases. Clin Rheumatol 1994; 13: 289-92.

76. Roig López De Los Mozos A, Calvo Catalá J, Hortelano Martínez E, Liñana Santafe JJ, González-Cruz Cervellera MI, Ballester Belda JE. Tratamiento con inmunosupresores en la arteritis de la temporal de larga evolución. An Med Interna (Madrid) 1992; 9: 181-2.

77. Betz M, Fox BS. Prostaglandin E2 inhibits production of Th1 lymphokines but not of Th2 lymphokines. J Immunol 1991; 146: 108-13.

78. González-Gay MA, Blanco R, Abraira V, et al. Giant cell arteritis in Lugo, Spain, is associated with low longterm mortality. J Rheumatol 1997; 24: 2171-6.

79. Nordborg E, Bengtsson BA. Death rates and causes of death in 284 consecutive patients with giant cell arteritis confirmed by biopsy. BMJ 1989; 229: 549-50.

80. Nesher G, Sonnenblick M, Friedlander Y. Analysis of steroid related complications and mortality in temporal arteritis: a 15-year survey of 43 patients. J Rheumatol 1994; 21: 1283-6.

81. Speed CA, Haslock I. Polymyalgia rheumatica, temporal arteritis and malignancy. Postgrad Med J 1995; 71: 500-2

82. Haga H-J, Eide GE, Brun J, Johansen A, Langmark F. Cancer in association with polymyalgia rheumatica and temporal arteritis. J Rheumatol 1993; 20: 1335-9. 